\title{
PRIMERA CITA DE STEPHANODISCUS AGASSIZENSIS HAKANSSON \& KLING (BACILLARIOPHYCEAE) EN CHILE
}

\section{FIRST RECORD OF STEPHANODISCUS AGASSIZENSIS HAKANSSON \& KLING (BACILLARIOPHYCEAE) IN CHILE}

\author{
Patricio Rivera ${ }^{1}$, Fabiola Cruces $^{1} \&$ Irma Vila $^{2}$
}

\begin{abstract}
RESUMEN
Se señala por primera vez para Chile a Stephanodiscus agassizensis Hakkansson \& Kling (Stephanodiscaceae). La especie fue encontrada en una muestra proveniente

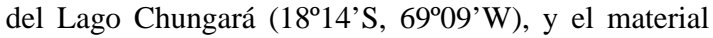
analizado está depositado en la Colección Diatomológica de la Universidad de Concepción, Chile. Fotomicrografías obtenidas en los microscopios fotónico y electrónico de barrido muestran las principales características morfológicas de este taxón.
\end{abstract}

Palabras Claves: Bacillariophyceae, diatomeas, Lago Chungará, nueva cita.

\section{INTRODUCCION}

Stephanodiscus Ehrenberg, género tipo de la familia Stephanodiscaceae Makarova (in Glezer \& Makarova 1986), presenta una amplia distribución mundial, siendo muy común en el plancton de lagos, ríos y de grandes masas de aguas dulces continentales. Sus especies se caracterizan por presentar valvas circulares, concéntricamente onduladas o planas, con un anillo de notorias espinas en la unión de la cara valvar con el manto, aréolas con la criba

${ }^{1}$ Departamento de Botánica, Universidad de Concepción, Casilla 160-C, Concepción, Chile. Email: privera@udec.cl

${ }^{2}$ Laboratorio de Limnología, Departamento de Ciencias Ecológicas, Universidad de Chile, Casilla 653, Santiago. E-mail: limnolog@abello.dic.uchile.cl

\begin{abstract}
Stephanodiscus agassizensis Hakansson \& Kling (Stephanodiscaceae) is reported for the first time from Chilean waters. The species was found in a sample collected in Lake Chungará $\left(18^{\circ} 14^{\prime} \mathrm{S}, 69^{\circ} 09^{\prime} \mathrm{W}\right)$, and the material is deposited in the Diatom Collection of the University of Concepción, Chile. Photographs taken under light and scanning electron microscopes show the principal morphological features of this taxon.
\end{abstract}

Keywords: Bacillariophyceae, diatoms, Lake Chungará, new record.

interna con aspecto de domo tanto en la cara valvar como en el manto y dispuestas en fascículos radiales, uno o más procesos labiados marginales provistos de un definido tubo exterior, y procesos reforzados dispuestos en el manto y sobre la cara valvar en posición iso o heterotópica.

Cyclostephanos, Cyclotella y Puncticulata son géneros afines pertenecientes a esta misma familia. Cyclostephanos fue propuesto por Round (1982) y validado en Theriot et al. (1987), y difiere de Stephanodiscus principalmente por presentar aréolas en fascículos sobre la cara valvar que se continúan en alvéolos en el manto, y procesos labiados marginales carentes de tubo exterior, situados debajo de una espina. En Cyclotella (Kützing) Brebisson 1838 existe un área central hialina y alvéolos marginales, y en Puncticulata, género recientemente propuesto por Hakansson (2002), existen alvéolos marginales y la parte central de la valva presenta aréolas y procesos reforzados, o sola- 
mente procesos reforzados o solamente aréolas, y los procesos labiados se sitúan sobre la cara valvar.

En Chile, el género Stephanodiscus fue señalado para Calbuco y Río Pascua por Krasske (1939, S. hantzschii Grunow), y para la Laguna Chica de San Pedro, Concepción, por Urrutia et al. (2000) como Stephanodiscus sp. Según Hakansson (2002) S. astraea (Ehr.) Grunow, señalado por diversos autores para lagos del sur de Chile (Rivera 1983), es un sinónimo de Cyclotella astraea (Ehr.) Kützing.

El análisis diatomológico de muestras de fitoplancton y de sedimento recolectadas en el Lago Chungará en distintos meses y años reveló la presencia, en sólo una de ellas (22.01.1999, 0-7 m. profundidad), de Stephanodiscus agassizensis Hakansson \& Kling (1989). Según nuestra información, además de la localidad tipo en Canadá y de la cita de Kling (1998) para ese mismo país, S. agassizensis también ha sido señalado para diversos ríos, lagos y embalses de Europa (Genkal 1993, Kiss \& Genkal 1993 y Findlay et al. 1998) y de Estados Unidos (Leland \& Berkas 1998, como S. cf. agassizensis). No tenemos antecedentes de que este taxón haya sido señalado anteriormente para Sudamérica. Según Hakansson \& Kling (1989) es una especie dominante de aguas ricas en nutrientes y con limitación de luz. Genkal (1993) señala que se desarrolla entre la primavera y el otoño, en aguas con temperatura entre 10,2 y $24^{\circ} \mathrm{C}, \mathrm{pH} 7,6-8,2$ y salinidad sobre 5 psu.

El Lago Chungará es el más austral (18 $14^{\prime}$ 'S, 6909'W) y de mayor altura de los lagos altoandinos (4.520 ms.n.m). Su cuenca tiene una superficie de $260 \mathrm{~km}^{2}$ y alcanza una profundidad máxima de $34 \mathrm{~m}$. Es moderadamente salino, puede ser clasificado como meso eutrófico (Sanzana \& Thoman 1985, Muhlhauser et al. 1995), y presenta una fuerte inhibición de la actividad fotosintética producida por la luz, lo cual influye en una estratificación notoria de las microalgas.

El material estudiado se encuentra depositado en la Colección Diatomológica de la Universidad de Concepción, bajo el número DIATCONC M-3200. Para la eliminación de la materia orgánica de los frústulos se utilizó el método descrito por Hasle \& Fryxell (1970). La especie fue estudiada mediante técnicas de microscopía fotónica (Fotomicroscopio III, Zeiss) y electrónica de barrido (ETEC Autoscan U-1). La terminología usada en este estudio es la propuesta por Anonymous (1975), por Ross et al. (1979) y por Hakansson (2002).

\section{DESCRIPCION}

\section{Stephanodiscus agassizensis Hakansson \&} Kling, p. 283, Figs. 56-69 (1989)

Figs. 1-18

Referencias: Krammer \& Lange-Bertalot 1991, p. 69, Figs. 71/6, 72/1-2b; Genkal 1993, p. 46, Figs. 1-34; Hakansson 2002, p. 35, Figs. 97-103. Localidad tipo: Red River, Manitoba, Canadá.

Valvas circulares, $12-28 \mu \mathrm{m}$ de diámetro (Figs. 1-3, 6, 9, 11, 13) . Cada cingulum está compuesta por 4-6 bandas abiertas, provistas de lígula, formando con las aberturas una línea oblicua, dextrógira (Fig. 4). La valvocópula es ancha y presenta una línea de pequeñas perforaciones alargadas, 7-8 en $1 \mu \mathrm{m}$, ubicada en la zona submarginal del lado advalvar (Fig. 5). Las pleuras no están estructuradas y disminuyen gradualmente de ancho a medida que se alejan de la valva (Figs. 4-5). En cada frústulo, una valva presenta la parte central notoriamente convexa, pero en la valva opuesta es cóncava (Fig. 6). Aréolas radiales, formando fascículos uniseriados en la parte central de la valva pero bi o triseriados en la unión de la cara valvar con el manto, 8-10 (11) en $10 \mu \mathrm{m}$ (Figs. 1-3, 6-14, 17-18). Interfascículos levemente elevados sobre la superficie valvar, 0,3-0,6 $\mu \mathrm{m}$ de ancho (Figs. 7-8, 14). Procesos reforzados con disposición heterotópica en las valvas, con abertura externa algo engrosada (Figs. 7, 17, flechas). En la valva convexa estos procesos se ubican en la zona elevada, subcentrales, en número de $1-3$, a veces 4 (Figs. 6-7 flechas, 9), cada uno con dos poros satélites (Fig. 10). En las valvas cóncavas se encuentran más cerca de la unión con el manto valvar, en número de 2-3, excepcionalmente 5 (Figs. 6, 11, flechas), cada uno provisto de 2 (Fig. 12) o muy raramente de 3 poros satélites (Fig. 18). Un anillo de espinas, a veces con el extre- 
mo dividido (Fig. 14), se encuentra en la unión de la cara valvar con el manto, situadas en cada interfascículo (Figs. 3, 13) o cada 3-4 interfascículos (Figs. 1-2, 8, 14). Manto valvar alto, con líneas diagonales de pequeñas aréolas, 4-5 en $1 \mu \mathrm{m}$. Un anillo de procesos reforzados, 3-4 (5) en $10 \mu \mathrm{m}$, se ubica en el manto valvar a corta distancia debajo de las espinas, externamente son algo engrosados (Figs. 4,8 ) e internamente llevan 3 poros satélites (Fig. 15). Existe sólo un proceso labiado en cada valva, ubicado entre el anillo de espinas; el tubo exterior presenta un largo similar al de las espinas (Figs. 16, 17 flecha), interiormente es sésil y con el labium en posición algo oblicuo (Figs. 11-12, 18).
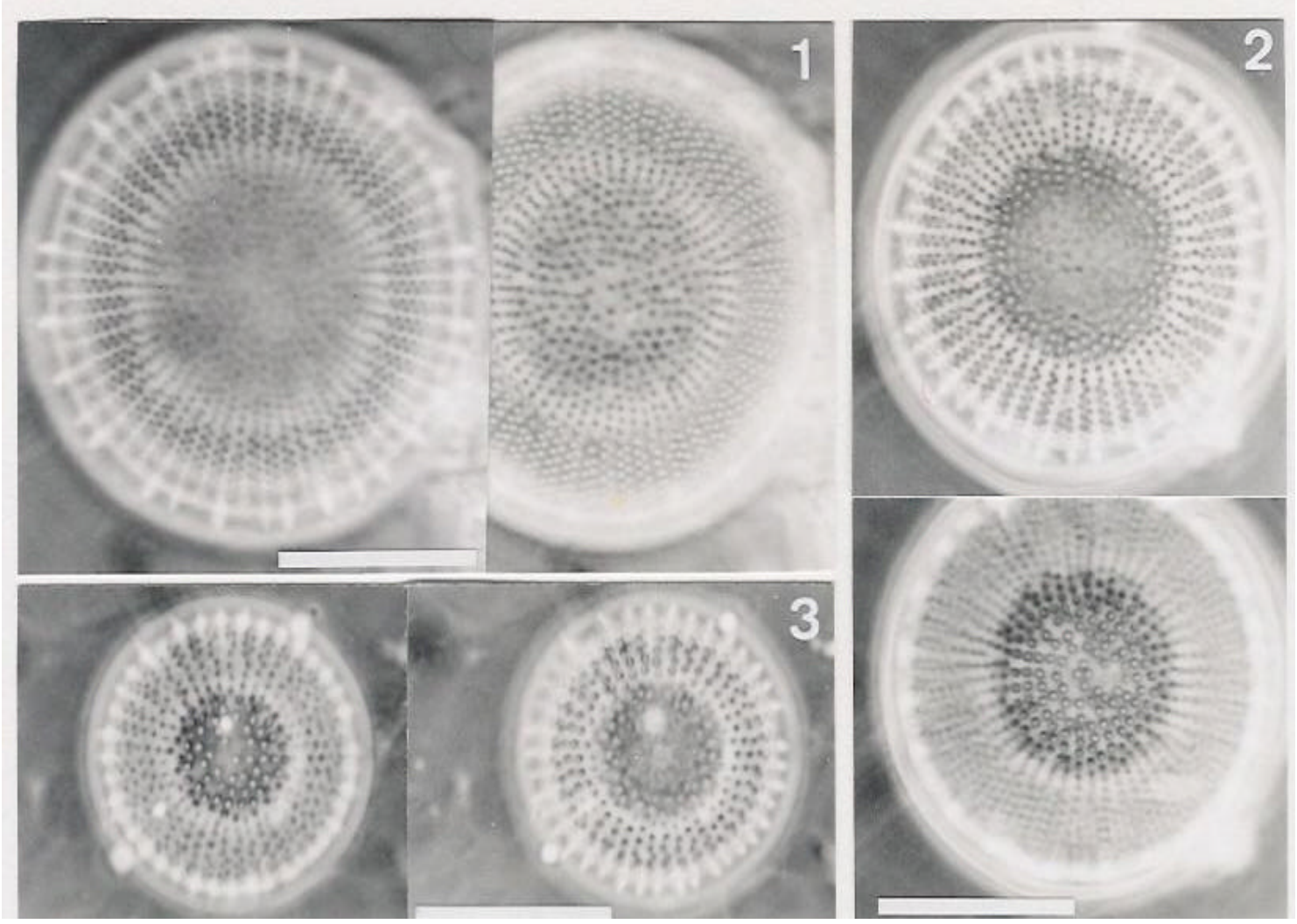

Figs. 1-3. Stephanodiscus agassizensis. LM. Escala $=10 \mu \mathrm{m}$. Foco a diferentes niveles para mostrar los fascículos de aréolas (uniseriados en el centro de la valva y biseriados en la unión con el manto valvar) y las espinas marginales. $\mathrm{LM}=$ microscopía fotónica. 


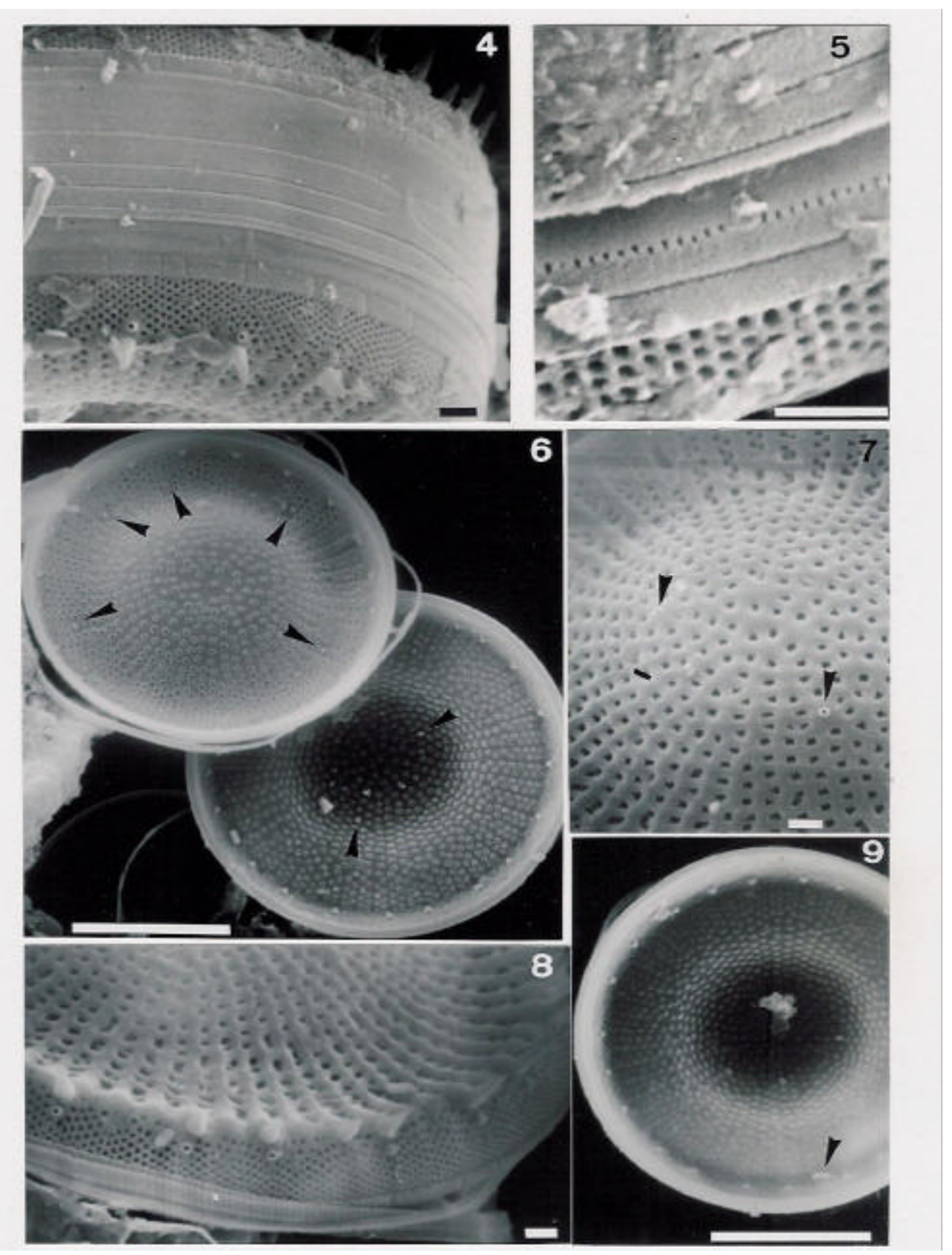

FIGs. 4-9. Stephanodiscus agassizensis. SEM. Escala $1 \mu \mathrm{m}$, excepto Figs. 6, 9= $10 \mu \mathrm{m}$. Fig. 4. Epicingulum formado por una ancha valvocópula y 4 pleuras; aberturas en posición dextrógira. Manto valvar alto con pequeñas aréolas; procesos reforzados cerca de las espinas, con abertura externa engrosada. Fig. 5. Aréolas del manto valvar, valvocópula con línea de pequeñas perforaciones y pleuras no estructuradas. Fig. 6. Vista interior de valvas cóncava y convexa mostrando los fascículos de aréolas, la posición heterotópica de los procesos reforzados valvares (flechas) y la posición de los procesos reforzados del manto. Fig. 7. Vista exterior del centro de una valva convexa mostrando la abertura externa engrosada de dos procesos reforzados. Interfascículos algo elevados sobre la superficie valvar. Fig. 8. Manto valvar alto, con pequeñas aréolas en líneas diagonales; procesos reforzados engrosados, a corta distancia de las espinas. Interfascículos elevados sobre la superficie valvar. Fig. 9. Vista interna de una valva convexa. Note la posición subcentral de los procesos reforzados valvares y del proceso labiado en la unión de la valva con el manto valvar (flecha). SEM= microscopía electrónica de barrido. 


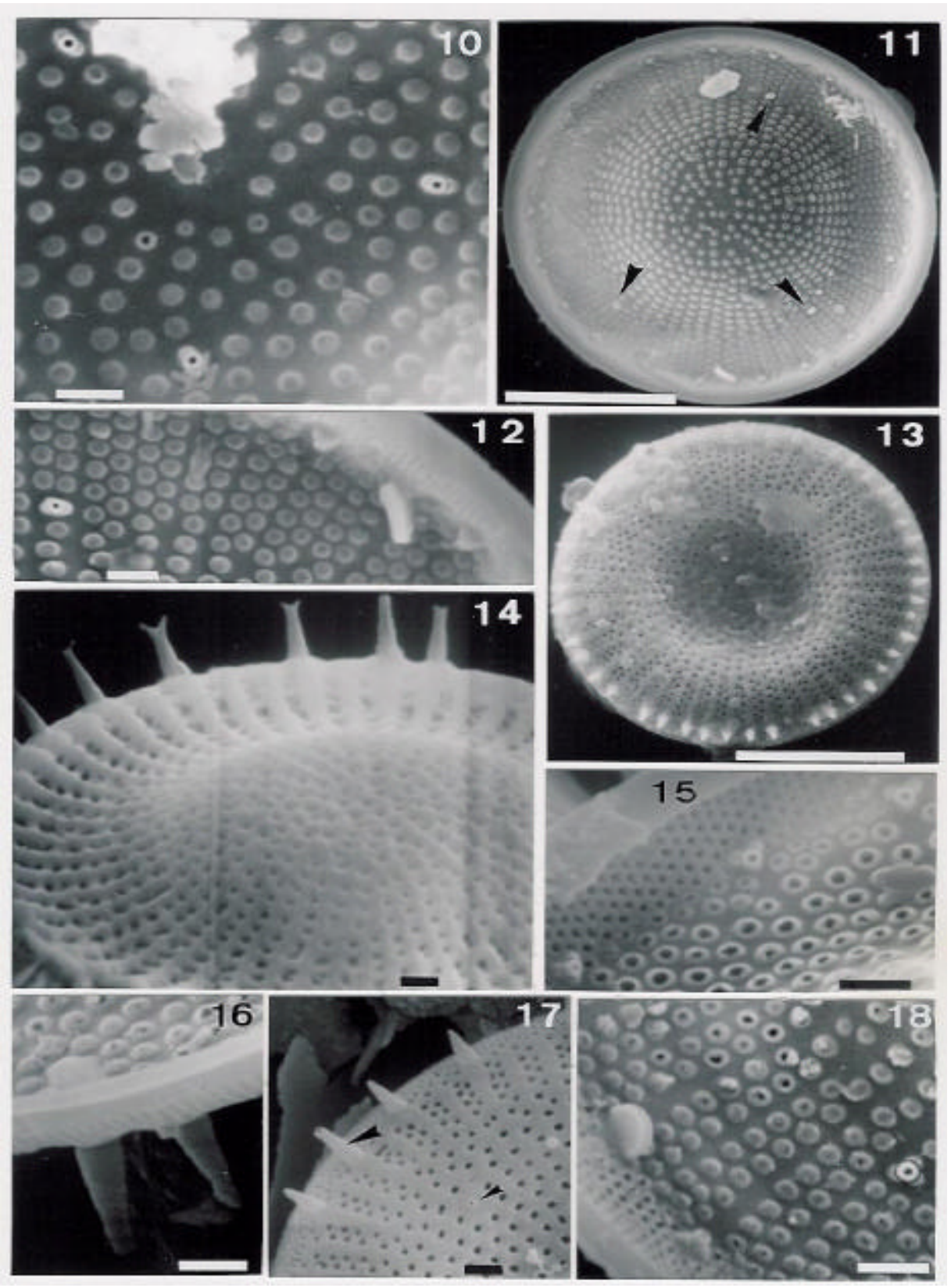

Figs. 10-18. Stephanodiscus agassizensis. SEM. Escala $1 \mu \mathrm{m}$, excepto Figs. 11, $13=10 \mu \mathrm{m}$. Fig. 10. Procesos reforzados de una valva convexa, cada uno con dos poros satélites. Fig. 11. Vista interna de una valva cóncava mostrando la posición submarginal de los procesos reforzados valvares (flechas), los fascículos de aréolas, la posición oblicua del proceso labiado y los procesos reforzados del manto valvar. Fig. 12. Proceso reforzado valvar con dos poros satélites y proceso labiado oblicuo, sésil. Fig. 13. Vista exterior de una valva cóncava provista de espinas marginales en cada interfascículo. Fig. 14. Vista exterior de una valva convexa. Interfascículos elevados sobre la superficie valvar; espinas distanciadas entre sí, algunas bifurcadas. Fig. 15. Manto valvar con pequeñas aréolas y procesos reforzados con tres poros satélites. Fig. 16. Tubo exterior del proceso labiado de igual largo a las espinas. Fig. 17. Valva cóncava mostrando la abertura externa de un proceso reforzado valvar (flecha pequeña), y el tubo exterior del proceso labiado (flecha grande). Fig. 18. Vista interior de una valva cóncava. Fascículos de aréolas bi o triseriados, un proceso reforzado valvar provisto de tres poros satélites y parte interna del proceso labiado, sésil y oblicuo. SEM= microscopía electrónica de barrido. 


\section{DISCUSION}

Excepto por el mayor diámetro valvar que alcanzan algunas células recolectadas en el Lago Chungará, las características morfológicas de este material coinciden con las descritas por Hakansson \& Kling para S. agassizensis. Sin embargo, el material ruso analizado por Genkal (1993) presentó diámetros entre 7 y $41,2 \mu \mathrm{m}$, valor muy superior al señalado en la descripción original. S. agassizensis es parecida a $S$. alpinus Hustedt, especie que presenta un menor número de procesos reforzados sobre la cara valvar, dispuestos en forma no heterotópica, las aréolas del manto valvar son de mayor tamaño, y los procesos reforzados allí distribuidos poseen solamente dos poros satélites. S. galileensis Hakansson \& Ehrlich, otra especie cercana, presenta un manto valvar de menor altura y estructurado en forma diferente, un proceso labiado pedunculado y pequeñas espinas marginales variables en número o inexistentes en algunos casos. Las características morfológicas y morfométricas de estas especies se resumen en la Tabla I.

TABla I. Principales características de S. agassizensis y de otras especies afines.

\begin{tabular}{|c|c|c|c|c|}
\hline & $\begin{array}{l}\text { S. agassizensis } \\
\text { Presente estudio }\end{array}$ & $\begin{array}{c}\text { S. agassizensis } \\
\text { Hakanss. \& Kling1989 } \\
\text { Hakansson } 2002 \\
\end{array}$ & $\begin{array}{c}\text { S. alpinus } \\
\text { Hakanss. \& Kling1989 } \\
\text { Hakansson } 2002 \\
\end{array}$ & $\begin{array}{l}\text { S. galileensis } \\
\text { Hakansson } 2002\end{array}$ \\
\hline Diámetro $(\mu \mathrm{m})$ & $12-28$ & $10-18,5$ & $7,5-33$ & $32-48$ \\
\hline $\begin{array}{l}\text { Líneas aréolas por } \\
\text { fascículo }\end{array}$ & $1-3$ & $1-3$ & $\begin{array}{l}1-2(3) \text {, desorga- } \\
\text { nizadas centro }\end{array}$ & $1-3$ \\
\hline $\begin{array}{l}\text { Ancho interfascículos } \\
(\mu \mathrm{m})\end{array}$ & $0,3-0,6$ & --- & --- & $0,3-0,6$ \\
\hline $\begin{array}{l}N^{o} \text { fascículos en manto } \\
(10 \mu \mathrm{m})\end{array}$ & $8-10(12)$ & --- & --- & --- \\
\hline $\begin{array}{l}\text { Posición procesos } \\
\text { reforzados valvares }\end{array}$ & heterotópica & heterotópica & no heterotópica & heterotópica \\
\hline $\begin{array}{l}\mathrm{N}^{\circ} \text { procesos reforzados } \\
\text { valvares y poros } \\
\text { satélites }\end{array}$ & $1-3(5) / 2(3)$ & $1-3 / 2$ & $0-1(2) / 3$ & varios / 2 \\
\hline $\begin{array}{l}\mathrm{N}^{\circ} \text { procesos reforzados } \\
\text { manto y poros satélites }\end{array}$ & $3-4(10 \mu \mathrm{m}) / 3$ & cada $2^{\mathrm{a}}-4^{\mathrm{a}}$ espina / 3 & $---/ 2$ & $---/ 3$ \\
\hline Proceso labiado & 1, sésil, oblicuo & 1, sésil, oblicuo & 1, sésil & 1 , pedunculado \\
\hline $\begin{array}{l}\text { Líneas aréolas en manto } \\
(1 \mu \mathrm{m})\end{array}$ & $4-5$ & --- & --- & --- \\
\hline Espinas & $\begin{array}{l}\text { en cada interfas- } \\
\text { cículo o c } / 3^{\circ}-4^{\circ} \text {, } \\
\text { algunas bifurcadas }\end{array}$ & $\begin{array}{l}\text { en cada interfas- } \\
\text { cículo o c } / 3^{\circ}-4^{\circ} \text {, } \\
\text { algunas bifurcadas }\end{array}$ & $\begin{array}{l}\text { en cada interfas- } \\
\text { cículo o irregulares }\end{array}$ & 0 -varias \\
\hline
\end{tabular}


Diversos autores han dado a conocer sus observaciones acerca de la variabilidad de algunos caracteres morfológicos presentes en Stephanodiscus. Entre ellos se encuentran el número de procesos reforzados valvares (Genkal 1993, encontró que en S. agassizensis variaban entre 1-9), el número de líneas de aréolas en cada fascículo, el ancho de los interfascículos y el número de aréolas sobre la valva, caracteres que pueden variar durante el ciclo de vida de la especie (Theriot \& Stoermer 1982, 1984; Hakansson 2002). Por otra parte, Hakansson (comunicación personal) considera que la posición de los procesos reforzados y el número de poros satélites, presentes tanto en la cara valvar como en el manto, son buenos caracteres morfológicos que permiten diferenciar a las especies. Sin embargo, en uno de los numerosos individuos de S. agassizensis observados en el material chileno, un proceso reforzado valvar de una valva cóncava presentó tres poros satélites en lugar de dos, revelando que este carácter también puede ser variable.

Muy poca atención se ha puesto a la estructura del cingulum de Stephanodiscus, en el caso particular de $S$. agassizensis, ésta no había sido descrita anteriormente. Round (1970) señala algunas características de las bandas de este género, pero la información existente para la mayoría de los taxa descritos es prácticamente nula. No debemos olvidar que en otros géneros, por ejemplo Thalassiosira, los caracteres morfológicos presentes en el cingulum son de gran importancia en la diferenciación de las especies.

En la muestra estudiada, S. agassizensis se presentó acompañado de Aulacoseira granulata (Ehr.) Simonsen, Cyclotella meneghiniana Kützing, Cyclostephanos andinus (Theriot, Carney \& Richerson) Tapia, Theriot, Fritz, Cruces \& Rivera, Cocconeis placentula Ehr., Asterionella formosa Hassall, y de diversas clorofíceas y cianobacterias.

\section{AGRADECIMIENTOS}

Los autores agradecen a la Dra. Hannelore Hakansson por su buena disposición para analizar las fotografías obtenidas del material chileno y por entregarnos importantes comentarios sobre la especie determinada y del género Stephanodiscus. Agradecemos también la ayuda prestada por el personal del Laboratorio de Microscopía Electrónica de la
Universidad de Concepción, en especial de los señores Hugo Pacheco, Raúl Alarcón y Julio Pugin.

\section{BIBLIOGRAFIA}

AnONYmous. 1975. Proposals for a standardization of diatom terminology and diagnosis. Nova Hedwigia, Beihefte. 53: 323-354.

Brebisson, A. 1838. Considération sur les Diatomées et essai d'une classification des genres et des espéces appartenant á cette famille. Falaise, $20 \mathrm{pp}$.

Findlay, D.L., H.J. Kling, H. Ronicke \& W.J. Findlay. 1998. A paleolimnological study of eutrophied Lake Arendsee (Germany). Journal of Paleolimnology 19: 41-54.

GenKaL, S.I. 1993. Large-celled undulated species of the genus Stephanodiscus Ehr. in USSR reservoirs: morphology, ecology and distribution. Diatom Research 8: 45-64.

Glezer, Z.I. \& I.V. MaKarova. 1986. New Order and Family of Diatoms (Bacillariophyta). Botaniceskji zurnal (Moscow \& Leningrad). 71: 673-676.

HaKansson, H. 2002. A compilation and evaluation of species in the general Stephanodiscus, Cyclostephanos and Cyclotella with a new genus in the family Stephanodiscaceae. Diatom Research 17: 1-139.

HaKansson, H. \& H. KLing. 1989. A light and electron microscope study of previously described and new Stephanodiscus species (Bacillariophyceae) from central and Northern Canadian lakes, with ecological notes on the species. Diatom Research 4: 269-288.

Hasle, G.R. \& G.A. Fryxell. 1970. Diatoms: cleaning and mounting for light and electron microscopy. Transactions of the American Microscopical Society 89: 469-474.

KISS, K.T \& S.I GENKAL. 1993. Winter blooms of centric diatoms in the River Danube and in its side-arms near Budapest (Hungary). Hydrobiologia 269/ 270: 317-325.

KLING, H.J. 1998. A summary of past and recent plankton of Lake Winnipeg, Canada, using algal fossil remains. Journal of Paleolimnology 19: 297307.

Krammer, K. \& H. Lange-Bertalot. 1991. Bacillariophyceae. 3 Teil: Centrales, Fragilariaceae, Eunotiaceae. In: Süsswasserflora von Mitteleuropa, Band 2/3 (eds Ettl, H., Gerloff, J., Heynig, H. \& D. Mollenhauer), 576 pp.

Krasske, G. 1939. Zur Kieselalgenflora Südchiles. Archives of Hydrobiology. 35: 349-468.

LELAND, H.V. \& W.R. BeRKAS. 1998. Temporal variation in plankton assemblages and physicochemistry of Devils Lake, North Dakota. Hydrobiologia 377: 57-71.

Mühlhauser, H.A., N. Hrepic, P. Mladinic, V. 
Gayana Bot. 59(2), 2002

Montecino \& S. Cabrera. 1995. Limnological features of the Andean Lake Chungará, northern Chile. Revista Chilena de Historia Natural 68: 341-349.

Rivera, P. 1983. A Guide for References and Distribution for the Class Bacillariophyceae in Chile between $18^{\circ} 28^{\prime} \mathrm{S}$ and $58^{\circ} \mathrm{S}$. Bibliotheca Diatomologica, Band 3, 386 pp.

Ross, R., E.J. Cox, N.I. Karayeva, D.G. Mann, T.B.B. Paddock, R. Simonsen \& P.A. Sims. 1979. An Amended Terminology for the Siliceous Components of the Diatom Cell. Nova Hedwigia, Beihefte 64: 513-533.

Round, F.E. 1970. The Delineation of the Genera Cyclotella and Stephanodiscus by Light Microscopy Transmission and Reflecting Electron Microscopy. Nova Hedwigia, Beihefte 31: 591-604.

Round, F.E. 1982. Cyclostephanos. A new genus within the Sceletonemaceae. Archiv Protistenk 125: 323329.

Sanzana, J. \& R. Thoman. 1985. Estudio limnológico en el Lago Chungará. Departamento de Investigación y Desarrollo Científico, Universidad de Tarapacá $21 \mathrm{pp}$.

Theriot, E. \& E.F. Stoermer. 1982. Observation on North American populations of Stephanodiscus (Bacillariophyceae) species attributed to Friedrich Hustedt. Amererican Microscopy Society 101: 368-374.

Theriot, E. \& E.F. Stoermer. 1984. Principal Component Analysis of variation in Stephanodiscus rotula and $S$. niagarae (Bacillariophyceae). Systematic Botany 9: 53-59.

Theriot, E., H. Hakansson, J.P. Kociolek, F.E. Round \& E.F. Stoermer. 1987. Validation of the centric diatom genus name Cyclostephanos. British Phycological Journal 22: 345-347.

Urrutia, R., K. Sabbe, F. Cruces, K. Pozo, J. Becerra, A. Araneda, W. Vyverman \& O. Parra. 2000. Paleolimnological studies of Laguna Chica de San Pedro (VIII Region): Diatoms, hydrocarbons and fatty acid records. Revista Chilena Historia Natural 73: 717-728.

Fecha de recepción: 16.07.02

Fecha de aceptación: 10.10.02

Fecha de publicación: Marzo de 2003. 